\title{
Variación en atributos anatómicos asociados a la conducción y almacenamiento de agua, en tres poblaciones de Maihuenia poeppigii (Otto ex Pfeiff.) K. Schum. (Cactaceae) en un gradiente altitudinal
}

\author{
Variation in anatomical traits associated with water conduction and storage in three \\ populations of Maihuenia poeppigii (Otto ex Pfeiff.) K. Schum. (Cactaceae) in an \\ altitudinal gradient
}

\author{
Taís Cabral ${ }^{1}$, Iván M. Quezada ${ }^{2 *}$ \& Alfredo Saldaña ${ }^{2}$ \\ ${ }^{1}$ Instituto Estadual do Ambiente. Av. Felíciano Sodré, 8, Centro, Niterói, Rio de Janeiro. Brasil. \\ 2Departamento de Botánica. Universidad de Concepción Casilla 160-C. Concepción. Chile. \\ *iquezada@udec.cl
}

\begin{abstract}
RESUMEN
La variabilidad en atributos anatómicos de las plantas podría estar relacionada con las condiciones del medio donde ellas se desarrollan (bajas temperaturas, estrés hídrico, corta estación de crecimiento), y esta variabilidad reflejaría la capacidad de una especie de establecerse en hábitats que difieren en la disponibilidad de estas condiciones. Maihuenia poeppigii (Otto ex Pfeiff.) K. Schum. no sólo es una de las especies de la familia Cactaceae con distribución más austral de América, sino que además presenta poblaciones creciendo en un amplio rango altitudinal (aprox. desde 15 hasta $2.600 \mathrm{~m}$ s.n.m.), en donde el clima varía notablemente. Este estudio evalúa la variabilidad en atributos anatómicos del tallo, relacionados con la conducción del xilema (densidad, diámetro y conductividad hidráulica relativa de los vasos) y almacenamiento de agua (grado de suculencia del tallo y hojas), de tres poblaciones de M. poeppigii que se encuentran establecidas en un gradiente de altitud en la zona centro sur de Chile. Adicionalmente, se determinó la altura máxima de los cojines de forma de estimar el grado de exposición de estos al ambiente, considerando la variación en la altura de las plantas a mayores altitudes. El promedio de la densidad, diámetro y conductividad hidráulica relativa de los vasos varió significativamente entre las poblaciones de distinta altitud. Con el incremento en la altitud aumentó la densidad de vasos y disminuyó el diámetro y el aporte relativo de los mismos a la conductividad hidráulica. Asimismo, con el aumento en la altitud disminuyó la altura de los cojines y aumentó la suculencia de los tallos. La suculencia de las hojas no difirió entre las tres poblaciones. La variación encontrada en dichos atributos anatómicos refleja una estrategia diferencial en la utilización y almacenamiento del agua entre las poblaciones establecidas en el gradiente de altitud. Este patrón de respuestas funcionales puede explicar la capacidad de esta especie de cactácea de ocupar sitios inhóspitos que difieren en altitud.
\end{abstract}

Palabras clave: Gradiente, anatomía del xilema, suculencia, altitud, Chile central.

\begin{abstract}
Variability in anatomical stem traits could be related with the environmental conditions where plants develop (e.g. low temperatures, drought, short growing season), and this variability reflects the ability of a species to be established in habitats that differ in these conditions. Maihuenia poeppigii (Otto ex Pfeiff.) K. Schum is not only one of the Cactaceae species with the southernmost distribution of America, but also presents populations along a wide range of altitudes (approx. from 15 to 2,600 masl.), where the climate varies greatly. This study evaluates the variability in stem anatomical traits related to xylem conduction (density, diameter and relative hydraulic conductivity of vessels) and to water storage (degree of succulence of stem and leaves), in three populations of M. poeppigii that are established in an altitudinal gradient in the South-Central Chile. Additionally, the maximum height of the M. poeppigii cushions was determined as a measure of its exposure to the environment. Average density, diameter and relative hydraulic conductivity of vessels differed significantly among populations from different altitudes. While vessel density and succulence of the stems increased in populations located at higher altitudes in the gradient, diameter of the vessels and plant height decreased. The succulence of the leaves did not differ among the three surveyed populations. The variation found in these anatomical traits reflects a differential strategy in the use and storage of water between the populations established in the altitudinal gradient. This pattern of functional responses can explain the ability of this species to occupy sites that differ in altitude.
\end{abstract}

KEYwords: Gradient, xylem anatomy, succulence, altitude, Central Chile. 


\section{INTRODUCCIÓN}

La variación intraespecífica de atributos morfológicos y anatómicos es frecuente a lo largo de gradientes ambientales, ya sean latitudinales o altitudinales, como respuesta a las distintas condiciones ambientales existentes dentro de estos (Filella \& Peñuelas 1999, Rundel et al. 1994, Körner 2003). Dentro de las condiciones que presentan variaciones importantes a lo largo de gradientes de altitud destacan la radiación solar, la velocidad del viento, temperaturas, la longitud de la estación de crecimiento (Körner 2003, Cavieres et al. 2006) y la disponibilidad de agua para las plantas (Körner 1999). Se ha reportado que la variación en atributos anatómicos permite que las plantas tengan la capacidad de desarrollarse dentro de un determinado rango geográfico, e incluso expandirlo (Krebs 1985). Lo anterior sugiere que, dentro de un gradiente altitudinal por ejemplo, las especies cuyas poblaciones son capaces de tolerar fisiológicamente las presiones ambientales serán capaces de alcanzar mayores elevaciones (Körner 2003).

La variación de los atributos funcionales a lo largo de gradientes de altitud se relaciona con la presión selectiva del ambiente sobre un determinado carácter (Carlquist 1975, Körner 1999). Dentro de los atributos que varían reconocidamente dentro de los gradientes de altitud se encuentran el área y la densidad de los vasos conductores en la madera. Las plantas de crecimiento lento que se encuentran creciendo en sitios áridos o semiáridos (Hacke \& Sperry 2001, Ackerly 2004), o con bajas temperaturas (Zimmermann 1983), presentan una madera más densa (menor número de vasos por unidad de área) que aquellas que habitan en zonas más cálidas y húmedas, las que presentan un sistema conductor compuesto por vasos de mayor área (área transversal de cada uno de los vasos) y con una menor densidad (Carlquist \& Hoekman 1985, BarajasMorales 1985, Fahn et al. 1986, Lindorf 1994, Alves \& Angyalossy 2001). La densidad y área de los vasos tienen un efecto relevante en la conductividad de agua dentro del tallo (Preston 2006). Las especies que presentan vasos de mayor diámetro tienen una mayor cantidad de espacio disponible para transporte de agua por área en la sección transversal del tallo (Zimmermann 1983), aunque, por otro lado, los vasos más grandes son más vulnerables a la embolia por congelación, en particular en el xilema cuando el agua presenta baja presión (Davis et al. 1999). Otro atributo que puede variar con la altitud es la altura de las plantas (Körner 2003). Se ha reportado una disminución en la altura de las plantas a mayor altitud en diversos estudios (e.g. Rochow 1970, Neuffer \& Bartelheim 1989, Galen et al. 1991, Bauert 1996). Los individuos que habitan en zonas alpinas tienden a crecer muy apegados a la superficie del suelo, buscando evadir los efectos del fuerte viento y aprovechar la radiación del suelo para alcanzar temperaturas más altas que las del aire (Körner \& Larcher 1988, Körner 1999).
En Chile, con el incremento en la altitud, el factor determinante para las especies que crecen en forma de cojín es una combinación entre el estrés hídrico y las bajas temperaturas a las que están sometidas (Cabrera 2002). Se considera que el hecho de presentar un crecimiento a nivel del suelo les proporciona una mayor tolerancia, debido a que generan microclimas favorables bajo su dosel (Körner 2003, Cavieres et al. 2006). En las Cactáceas, la suculencia de los tallos se ha interpretado como un factor que amortigua la perdida de agua (Gibson 1973), asegurando la conducción y reduciendo el riesgo de cavitación en periodos de escasez (Mauseth 1993). Este grupo de plantas también se caracteriza por presentar un sistema vascular altamente especializado, donde los tejidos principales (xilema y floema) se producen entre la corteza y el interior de la médula (Terrazas \& Mauseth 2002). En la cactácea Maihuenia poeppigii, los conjuntos de xilema y floema se encuentran rodeando la médula (Mauseth 1999). El xilema está compuesto de vasos, células parenquimáticas y algunas traqueidas de banda ancha (Mauseth 1999). El floema se constituye de parénquima axial y radial, miembros de los tubos cribosos, las células acompañantes, y se caracteriza por tener pocos espacios intercelulares, debido a la gran cantidad de mucílago (Mauseth 1999). El tallo está constituido por una gruesa capa de tejido parenquimático y además carece de tejidos rígidos (inflexibles), como por ejemplo las células esclerenquimáticas. Esta característica anatómica permite a esta especie reducir los tallos (tamaño) al máximo frente a períodos de sequía, o hidratarse al máximo cuando el agua esté disponible (Mauseth 1999). Otra característica importante de la especie es su forma de crecimiento en cojín, caracterizada en otras especies de cojines de alta montaña similares por generar un microclima bajo su interior, el que amortigua las condiciones limitantes (fuerte viento, alta radiación solar, bajas temperaturas, baja disponibilidad de agua) que ocurren a mayores altitudes (Cavieres et al. 2002, Mauseth 1999, Körner 2003).

En la zona centro sur de Chile, M. poeppigii crece en distintas localidades en la provincia de Concepción, ocupando una franja que va entre $\operatorname{los} 36^{\circ} 00^{\prime}$ y los $38^{\circ} 30^{\prime} \mathrm{S}$, y desde aproximadamente los $30 \mathrm{~m}$ s.n.m. hasta el límite superior de la vegetación, a 2.600 m s.n.m., en la cordillera de los Andes (Mauseth 1999, Hoffmann \& Walter 2004). Se ha reportado que las precipitaciones disminuyen significativamente dentro de esta zona, al avanzar desde la costa hacia las mayores altitudes de los Andes (Mauseth 1999), al igual que las temperaturas medias, mientras la oscilacion térmica se hace mayor al alejarse de la costa. Por lo mismo, se podría considerar que dentro del rango de distribucion longitudinal de $M$. poeppigii existe un gradiente climático altitudinal marcado por variaciones en las precipitaciones y la temperatura. En cuanto a la variabilidad fenotípica de las poblaciones que se encuentran dentro del gradiente, hasta ahora sólo se ha reportado que los 
cojines de los sitios más cálidos de la costa y de la depresión intermedia son menos densos que los de la cordillera de los Andes, donde están cubiertos de nieve en el invierno (Kraus 1993). Además, se ha visto que el sistema de raíces superficiales de esta especie puede abarcar una superficie bastante grande (ca. $7 \mathrm{~m}$ de longitud), y que estas raíces son almacenadoras de agua y contienen mucílago (Leuenberger 1992). Sin embargo, hasta el momento no se han estudiado los atributos anatómicos que puedan reflejar diferencias funcionales entre las poblaciones de $M$. poeppiggi que se han establecido en zonas que difieren en altitud (Costa Depresión Intermedia- Cordillera de los Andes).

Considerando la presencia de poblaciones de $M$. poeppigii en un gradiente de altitud, a lo largo del cual se registran condiciones climáticas significativamente distintas, particularmente de precipitaciones y temperatura (Luebert \& Pliscoff 2006), el principal objetivo de este estudio fue determinar la variabilidad en atributos anatómicos relacionados con la conducción (densidad de vasos, diámetro de vasos y conductividad hidráulica relativa de los vasos) y almacenamiento de agua (suculencia del tallo y hojas) en tres poblaciones de $M$. poeppigii establecidas en dicho gradiente. Además, se evaluó si existen diferencias interpoblaciones en la magnitud de la relación entre los atributos de los vasos medidos, lo que reflejaría compromisos entre conducción (densidad de vasos en función del diámetro y conductividad hidráulica relativa de los vasos) y altitud de los sitios. Por último, se evaluó si existen diferencias en la altura máxima de los cojines, como una medida del grado de exposición al ambiente de estos.

\section{MATERIALES Y MÉTODOS}

\section{Sitios DE ESTUdio}

Se estudiaron tres poblaciones de $M$. poeppigii ubicadas a lo largo de un gradiente altitudinal localizado en la Región del Biobío. Debido a la falta de datos precisos y recientes de temperatura y precipitaciones para los puntos exactos de muestreo, se optó por recurrir a la base de datos climáticos Worldclim (Hijmans et al. 2005), para caracterizar de forma más precisa el gradiente climático. El sitio de menor altitud (Gomero), se ubica en la comuna de Hualqui, provincia de Concepción, a aproximadamente 39 m s.n.m. (37¹1'37's, $\left.72^{\circ} 47^{\prime} 24^{\prime \prime} \mathrm{O}\right)$. La precipitación anual es de $1.337 \mathrm{~mm}$, en tanto la temperatura media anual es de $13,4{ }^{\circ} \mathrm{C}$. Además, esta zona es reconocida por tener una escasa oscilación térmica (di Castri \& Hajek 1976). El segundo sitio de estudio (Cabrero) se localiza en la depresión intermedia, en la comuna de Cabrero, a 160 m s.n.m (3702'00'S, $72^{\circ} 24^{\prime} 00^{\prime}$ 'O), con una precipitación media anual de 1.094 mm y una temperatura media anual de $13,4{ }^{\circ} \mathrm{C}$. En esta región, el clima se caracteriza por presentar veranos largos y cálidos, e inviernos fríos (Hoffmann 1995). La depresión intermedia ha sido rellenada por materiales volcánicos de la Cordillera de los Andes, y constituye un área importante desde el punto de vista agrícola (Mourgues 1982). El tercer sitio de estudio está ubicado en el Parque Nacional Laguna del Laja (37²2'00"S, 71²6'00"O), a 1.208 m s.n.m. Presenta una precipitación media anual de $1.461 \mathrm{~mm}$ y una temperatura media anual de $8,6^{\circ} \mathrm{C}$. Sin embargo, aunque las precipitaciones anuales en este último sitio sean mayores que en los anteriores, la mayor parte de éstas ocurren en forma de nieve, lo que dificulta su acceso por parte de las plantas.

\section{MuestreO DE CAMPO}

En cada sitio se muestrearon al azar 30 cojines de $M$. poeppigii, seleccionando solo cojines de diámetro entre $70-80 \mathrm{~cm}$ (la media del diámetro de los cojines observados en cada población está en un rango de entre 67 y $84 \mathrm{~cm}$ ), presentes en sectores de similar nivel de perturbación antrópica, i.e. distancia mínima a borde de camino de 50 $\mathrm{m}$, en zonas abiertas, sin cobertura de dosel arbóreo o arbustivo, a distancia mínima de plantaciones forestales de $50 \mathrm{~m}$, esto último específicamente en el caso de los dos sitios más bajos. En cada uno de los cojines se seleccionaron al azar tres tallos principales cercanos al centro del cojín, bien desarrollados y en buen estado. En ellos se analizaron los siguientes atributos anatómicos: suculencia del tallo; suculencia de la hoja; densidad, diámetro, y conductividad hidráulica relativa de los vasos. Adicionalmente se midió la altura máxima de cada uno de los 30 cojines. En el análisis de los datos se consideró el promedio de los valores obtenidos para cada atributo como una medida del individuo (cojín). Los muestreos fueron realizados en el mes de octubre en las poblaciones de la costa (02/10/09) y la depresión intermedia (17/10/09), y a fines de la estación de primavera en las poblaciones de la cordillera de los Andes (06/11/09). El desfase en las fechas de los muestreos se debió a la necesidad de esperar el derretimiento de la nieve en las poblaciones de la cordillera de los Andes.

\section{MEDICIÓN DE LOS ATRIBUTOS ANATÓMICOS}

Las muestras fueron mantenidas frescas (adicionando agua y evitando exposición al sol) durante $24 \mathrm{~h}$ antes de ser procesadas. Para medir la densidad de vasos se seccionó un segmento de la base del tallo de cada individuo, y luego se seccionaron segmentos transversales del tallo, los cuales fueron observados de inmediato en el microscopio. En todos los casos, los cortes se realizaron cerca de la base del tallo, para estandarizar la toma de las muestras. Para analizar los cortes transversales se utilizó una cámara digital montada en un microscopio óptico, con lo cual se obtuvieron fotografías de los cortes, las que luego fueron analizadas con el programa SigmaScan (Systat Software Inc, USA). Se contabilizó el número de vasos del segmento transversal del tallo, en la región del metaxilema, el cual fue dividido por 
el área $\left(0,025 \mathrm{~mm}^{2}\right)$ ocupada por los vasos. El diámetro de los vasos fue analizado en 20 vasos por individuo (en cada población), en la región del metaxilema, a través del programa SigmaScan (Systat Software Inc, USA). Para el análisis se utilizó el promedio del diámetro de los 20 vasos, como medida del individuo (cojín). De acuerdo a la ley de HagenPoiseuille, el flujo en los vasos es proporcional al radio del lumen del vaso elevado a la cuarta potencia $\left(\mathrm{r}^{4}\right)$, lo cual se correlaciona con la conductividad hidráulica relativa de los vasos (Zimmermann 1983, Ewers \& Fisher 1989). Al elevar a la cuarta potencia, se obtiene la diferencia en conductividad (\%) al comparar con vasos de radios diferentes. Para medir la conductividad hidráulica relativa de los vasos, primero, y asumiendo una sección transversal circular de los vasos, se obtuvo el valor del radio de éstos (el cual se estima en base a la raíz cuadrada del área del lumen de los vasos dividida por PI $(\mathrm{R}=\sqrt{\mathrm{A}} / \pi)$, el cual fue elevado a la cuarta potencia $(r)^{4}$. La suculencia de los tallos y hojas se estimó a través del índice de suculencia, el cual se estima en base a la diferencia entre la masa fresca del tejido (tallo o hoja) y la masa seca del mismo, dividido por la masa fresca del tejido (Cornelissen et al. 2003). Para esto se utilizó una balanza electrónica de 3 decimales. La altura máxima de cada uno de los 30 individuos se midió cerca del centro de cada cojín utilizando una regla. Se obtuvieron tres mediciones de los puntos más altos del cojín, a través de la distancia entre el límite superior del tallo y la parte basal del mismo (Cornelissen et al. 2003). Para el análisis se utilizó el promedio de las tres medidas como medida del individuo (cojín).

ANÁLISIS DE LOS DATOS

Todas las variables medidas en este estudio (densidad y diámetro de vasos; conductividad hidráulica relativa de los vasos; suculencia tallo; suculencia hoja; altura máxima) presentaron distribución normal. Para determinar si existen diferencias significativas entre las tres poblaciones (Gomero, Cabrero y Laja) de $M$. poeppigii en el valor promedio de las variables anatómicas, se utilizó un análisis de varianza (ANOVA) de una vía y un test a posteriori (Tukey HSD) para determinar entre que pares de poblaciones existía la mayor diferenciación. Para comparar entre las poblaciones la magnitud de la relación entre pares de atributos medidos se utilizó un análisis de covarianza (ANCOVA), con la altitud/localidad como factor, diámetro de los vasos y conductividad hidráulica relativa de los vasos como variable respuesta, utilizando la densidad de vasos como covariable.

\section{RESULTADOS}

\section{VARIACIÓN EN ÁREA Y DENSIDAD DE LOS VASOS}

Las tres poblaciones de la especie $M$. poeppigii presentan diferencias significativas en el valor promedio del diámetro de los vasos (Tabla I). Las poblaciones que crecen en la cordillera de los Andes, (Laguna del Laja), y en la depresión intermedia (Cabrero) presentan vasos de menor diámetro que la población presente en la costa (Gomero) (Fig. 1A). El valor promedio de la densidad de vasos también difiere significativamente entre las tres poblaciones (Tabla I). Conforme aumentó la altitud, aumentó la densidad de vasos, siendo la población de Laguna del Laja (media= 1394,2 vasos $/ \mathrm{mm}^{2}$ ) la que presentó mayor densidad, seguida de la población de Cabrero (media=1387,5 vasos/ $\mathrm{mm}^{2}$ ) y Gomero (media=1209,2 vasos $/ \mathrm{mm}^{2}$ ) (Fig. 1B). Así mismo cuando se relacionó ambos atributos en un análisis de covarianza (ANCOVA), diámetro de los vasos en función de la densidad de vasos (covariable), se observó una tendencia negativa entre ambas variables (Fig. 2A). Sin embargo, a pesar de que el diámetro de los vasos fue diferente en relación a la densidad de vasos entre los sitios $(\mathrm{P}=0,0059)$, no se encontró una diferencia significativa en la interacción de ambas variables entre las tres poblaciones $(\mathrm{P}=0,1870)$ (Tabla II). El aporte relativo de los distintos tamaños de los vasos a la conductividad hidráulica presentó diferencia significativa entre las tres poblaciones $(\mathrm{P}=0,0011)$ (Tabla I). La conductividad hidráulica relativa de los vasos fue significativamente mayor en la población de Gomero $\left(\right.$ media $\left.=2,93 \times 10^{-11}\right)$, seguido de Cabrero (media=2,0 $\times 10^{-}$ $\left.{ }^{11}\right)$ y Laguna del Laja (media $=1,74 \times 10^{-11}$ ). De acuerdo con el test a posteriori (Tukey HSD), ambas poblaciones que se encuentran a mayor altitud en el gradiente (Cabrero y Laguna del Laja) diferirán significativamente de la población de Gomero en el valor promedio de la conductividad hidráulica relativa de los vasos (Fig. 3C). La interación entre ambos atributos (conductividad hidráulica relativa de los vasos en función de la densidad de vasos) presentó una tendencia negativa (Fig. 3B). Sin embargo ésta no resultó significativa para las tres poblaciones ( $\mathrm{P}=0,2375)$ (Tabla II).

\section{VARIACIÓN EN LA ALTURA MÁXIMA Y SUCULENCIA}

La altura máxima de los cojines de $M$. poeppigii también presentó un valor promedio altamente significativo entre las tres poblaciones $(\mathrm{P}<0001)$ (Tabla I). Los cojines de mayor altura fueron encontrados en la población de Gomero (media=16,3 cm), seguido de Cabrero (media=14,5 cm) y Laguna del Laja (media=11,0 cm). La mayor diferenciación se observó entre las poblaciones que se encontraban a una menor altitud (Gomero y Cabrero) y la población de Laguna del Laja (Fig. 3D). El valor promedio de la suculencia de los tallos difirió significativamente entre las tres poblaciones $(\mathrm{P}<0001)$ (Tabla I), al contrario de la suculencia de las hojas que no presentó diferencias significativas $(\mathrm{P}=0,2964)$ (Tabla I). Los tallos más suculentos fueron encontrados en la población de Cabrero (media=0,79 g), seguido de Laguna del Laja (media=0,74 g) y Gomero (media=0,71 g) (Fig. 3E). El test a posteriori (Tukey HSD) evidenció que los cojines de Gomero y Laguna del Laja difirieron mayormente de los cojines de Cabrero en el promedio de suculencia. 


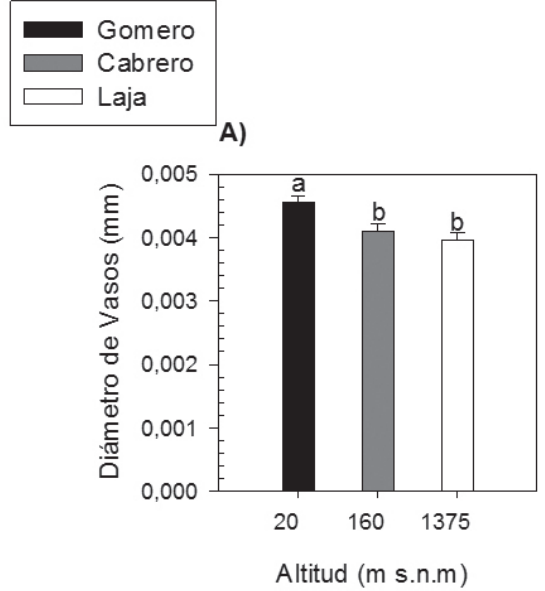

D)

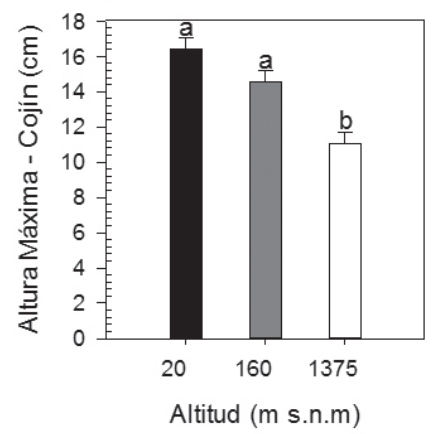

B)

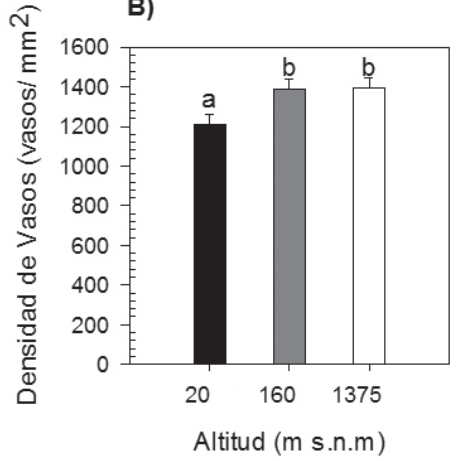

C)

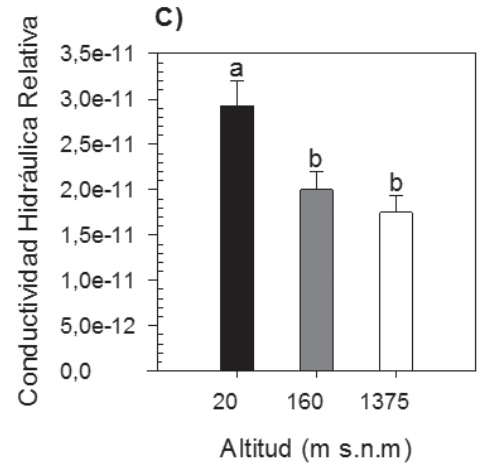

E)

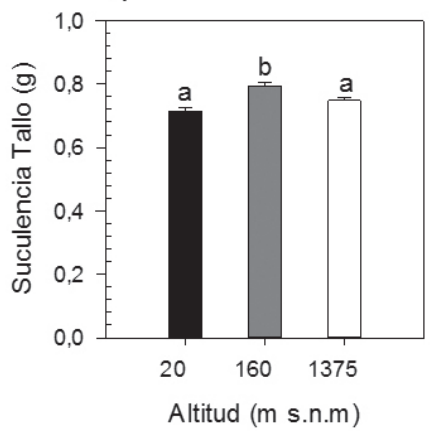

FIgURA 1. Valor promedio ( \pm EE) de los atributos anatómicos medidos en Maihuenia poeppigii en las tres poblaciones (Gomero, Cabrero y Laguna del Laja).

FIgURE 1. Mean values $( \pm \mathrm{SE})$ of anatomic attributes in Maihuenia poeppigii measured in the three populations (Gomero, Cabrero and Laguna del Laja).

TABLA I. Medias de los atributos anatómicos medidos en las tres poblaciones (Gomero, Cabrero y Laguna del Laja) de Maihuenia poeppigii. Asterisco $(*)$ indica diferencia significativa entre las poblaciones en ANOVA $(p<0,05)$.

TABLE I. Mean values of anatomical attributes measured in the three population (Gomero, Cabrero and Laguna del Laja) of Maihuenia poeppigii. Asterisk $\left(^{*}\right)$ indicates significant differences between populations in ANOVA $(\mathrm{p}<0.05)$.

\begin{tabular}{lccc}
\hline Atributos Anatómicos & Gomero & Cabrero & Laguna del Laja \\
\hline Diámetro de los vasos $(\mathrm{mm})$ & 0,00455 & 0,00409 & $0,00396\left(^{*}\right)$ \\
Conductividad Hidráulica Relativa & $2,93 \times 10^{-11}$ & $2,0 \times 10^{-11}$ & $1,74 \times 10^{-11}(*)$ \\
Densidad de vasos (vasos $\left./ \mathrm{mm}^{2}\right)$ & 1209,2 & 1387,5 & $1394,2\left(^{*}\right)$ \\
Altura Máxima $(\mathrm{cm})$ & 16,3 & 14,5 & $11,0\left(^{*}\right)$ \\
Suculencia de la Hoja $\left(\mathrm{g} / \mathrm{H}_{2} \mathrm{O}\right)$ & 0,77 & 0,79 & 0,77 \\
Suculencia del tallo $\left(\mathrm{g} / \mathrm{H}_{2} \mathrm{O}\right)$ & 0,71 & 0,79 & $0,74\left(^{*}\right)$ \\
\hline
\end{tabular}



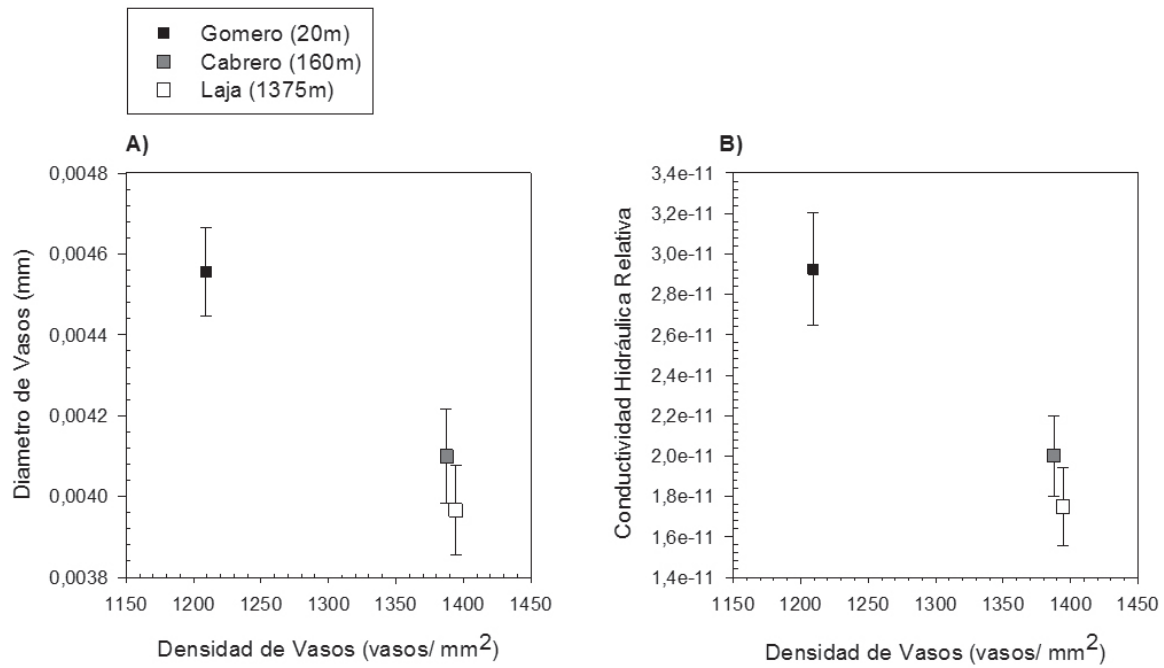

Figura 2. Diámetro y conductividad hidráulica relativa ( \pm EE) de los vasos de la especie Maihuenia poeppigii con la densidad de vasos como covariable.

Figure 2. Diameter and relative hydraulic conductivity ( $\pm \mathrm{SE}$ ) of vessels of the species Maihuenia poeppigii, with vessels density as covariate.

TABLA II. Análisis de covarianza (ANCOVA) del diámetro y conductividad hidráulica relativa de los vasos entre las tres poblaciones de Maihuenia poeppigii (Gomero, Cabrero y Laguna del Laja), con la densidad de vasos como covariable.

TABLE II. ANCOVA of diameter and relative hydraulic conductivity of vessels among the three populations of Maihuenia poeppigii (Gomero, Cabrero and Laguna del Laja), with the density of vessels as a covariate.

\begin{tabular}{lcccc}
\hline Atributo & df & ss & $f$ & $p$ \\
\hline DiÁmetro de LOS VASOs (mm) & & & & \\
Sitios & 2 & $3,49239 \mathrm{e}-6$ & 5,490 & 0,005 \\
Densidad de los vasos & 1 & $1,98636 \mathrm{e}-6$ & 6,245 & 0,014 \\
Sitios*Densidad vasos & 2 & $1,09 \mathrm{e}-6$ & 1,713 & 0,187 \\
& & & & \\
ConductividAD HIDRÁULICA RELATIVA & & & & 0,005 \\
Sitios & 2 & $1,3881 \mathrm{e}-21$ & 5,552 & 0,016 \\
Densidad de los vasos & 1 & $7,5298 \mathrm{e}-22$ & 6,024 & 0,237 \\
Sitios*Densidad vasos & 2 & $3,6618 \mathrm{e}-22$ & 1,464 & \\
\hline
\end{tabular}

\section{DISCUSIÓN}

Las variaciones de atributos funcionales a lo largo de gradientes de altitud ha sido abordada por una serie de estudios (Arnold \& Mauseth 1999, Cavieres et al. 2000, Cabrera 2002, Alberdi \& Donoso 2004, Souto et al. 2009). Las más estudiadas han sido las variaciones en los conductos xilemáticos (densidad, diámetro y conductividad hidráulica relativa de los vasos) (Mauseth 1999, Davis et al. 1999, Arias \& Terrazas 2001, Hacke \& Sperry 2001, Ladjal et al. 2005), en la altura (Cavieres et al. 2000, Körner 2003, Falster \& Westoby 2005), y en la suculencia de las plantas (Mauseth 1999, 2000, 2006) especialmente en especies que crecen en condiciones limitantes y/o a mayores altitudes (Körner 2003). Maihuenia poeppigii es una especie que se caracteriza por presentar adaptaciones anatómicas (vasos estrechos; tallos flexibles; gran capacidad de almacenar agua; crecimiento achaparrado, baja estatura), que le permiten tolerar las condiciones limitantes presentes en su rango de distribución (Mauseth 1999, Körner 2003). 
En este estudio se observa una variación en los atributos de los vasos de M. poeppigii, a medida que se incrementa la altitud. Las poblaciones ubicadas a mayores altitudes (Cabrero, $160 \mathrm{~m}$; Laguna del Laja, $1.375 \mathrm{~m}$ ) presentan vasos de menor diámetro a una mayor densidad, además de un menor aporte a la conductividad hidráulica en comparación a la población ubicada a menor altitud en el gradiente (Gomero, $20 \mathrm{~m}$ ) (Fig. 2). Tal patrón podría ser producto de las bajas temperaturas invernales $\left(-10{ }^{\circ} \mathrm{C}\right.$ a $\left.-20{ }^{\circ} \mathrm{C}\right)$ en los sitios más elevados (Mauseth 1999). Cuando la especie está sometida a temperaturas menores a $0{ }^{\circ} \mathrm{C}$, generalmente ocurre una congelación del espacio extracelular (apoplasto), causando deshidratación celular severa, daños físicos (Davis et al. 1999) y pudiéndose interrumpir el proceso de trasporte xilemático (Zimmermann 1983). Las adaptaciones que reducen al mínimo estos problemas han sido objeto de numerosos estudios (Sakai \& Larcher 1987, Carlquist \& Hoekman 1985, Davis et al. 1999, Arias \& Terrazas 2001, Maherali et al. 2004, Ladjal et al. 2005). Poseer un xilema con vasos de menor diámetro y en una mayor densidad puede implicar un menor riesgo de embolismo (Zimmermann \& Brown 1971, Ewers 1985, Davis et al. 1999, Pate \& Canny 1999, Sánchez-Díaz \& Aguirreolea 2000, Schnitzer \& Bongers 2002, Jiménez-Castillo \& Lusk 2013). Este fenómeno puede ocurrir principalmente debido a dos circunstancias: cuando la planta está sometida a condiciones de estrés hídrico, o a temperaturas inferiores a $0{ }^{\circ} \mathrm{C}$ (Gartner 1995, Lambers et al. 1998). Así, especies como $M$. poeppigii, que desarrollan un gran número de vasos pequeños, proporcionan una mayor estabilidad y resistencia al trasporte en el xilema, reduciendo el riesgo de embolismo (Mauseth 1999), y logrando alcanzar mayores altitudes. Esto es posible pues, aunque algunos vasos puedan cavitar al encontrarse la planta a temperaturas menores a $0{ }^{\circ} \mathrm{C}$, muchos todavía se encuentran llenos de agua y son capaces de mantener el proceso de conducción (Mauseth 1999). En contraste, vasos más anchos, como los encontrados en la población de menor altitud (Gomero - 39 m s.n.m), presentan la capacidad de sostener una mayor conductividad hidráulica (Gartner et al. 1990, Ewers 1985, Ewers \& Fisher 1991, Lambers et al. 1998), lo que a su vez puede facilitar la conductancia estomática y una mayor ganancia fotosintética de carbono (Santiago et al. 2004). Sin embargo, cuando estos individuos son sometidos a estrés hídrico o a temperaturas bajo $0{ }^{\circ} \mathrm{C}$, son más vulnerables a la cavitación (Preston 2006). A pesar de no haber diferencia significativa en la interacción de los atributos de los vasos (diámetro y conductividad hidráulica relativa en función de la densidad de vasos) entre las tres poblaciones (Tabla II), se puede observar la existencia de un compromiso entre los atributos: A medida que aumenta la densidad de vasos, disminuye el diámetro y el aporte relativo de los mismos a la conductividad hidráulica. Tal compromiso está vinculado a un aseguramiento en la conducción de agua en el xilema (Hacke \& Sperry 2001), en condiciones de estrés a mayores altitudes (Körner 2003). Futuros estudios deberían considerar otros atributos potencialmente importantes de la anatomía de los vasos, tales como el grosor de la pared de éstos y su longitud. Variaciones en estos atributos pueden conferir, bajo ciertas circunstancias, mayor resistencia mecánica, mayor resistencia al embolismo o una mayor eficiencia hidráulica (Kahn-Jetter et al. 2002, Jacobsen et al. 2005).

Otro atributo que varió con la altitud fue la altura máxima de los cojines, concordando con lo reportado por otros autores (Rochow 1970, Neuffer \& Bartelheim 1989, Galen et al. 1991, Bauert 1996, Körner 2003). Los cojines de mayor altura fueron encontrados en la población de Gomero, seguido de Cabrero y Laguna del Laja. Este resultado concuerda con lo propuesto previamente para especies que se establecen a gran altitud (Carlquist \& Hoekman 1985, Körner 2003), en donde la altura del cojín se vería influenciada por la presión que ejercería la nieve o hielo durante el periodo de invierno (Mauseth 1999). Además, la disminución de la temperatura a mayor altitud influye en el crecimiento de las plantas al tornar demasiado lenta la formación de nuevas células, aún en condiciones óptimas de ganancia de carbono (Körner 1999, Hoch et al. 2002, Körner 2003). Lo anterior sugiere que la formación de nuevas células es mucho más sensible a las bajas temperaturas que la fotosíntesis, por lo que el crecimiento vegetativo estaría restringido a bajas temperaturas, por la asignación de recursos dentro de la planta y no por una menor ganancia de carbono (Körner 2003). Por otro lado, el mantener una forma achaparrada facilita un mayor desacoplamiento térmico, lo que permite a la planta mantener una temperatura significativamente mayor dentro del cojín (Korner 2003, Mark 2008, Larcher 2012). Otro punto a considerar es que los individuos distribuidos a mayores elevaciones no disponen del mismo tiempo para crecer, debido al corto período de la estación de crecimiento (Cavieres et al. 2006). En este caso, los cojines de $M$. poeppigii establecidos en la zona costera presentarían un mayor tamaño pues no están sometidos a la misma presión que aquellos establecidos a mayor altitud.

La mayor suculencia fue encontrada en los individuos de la población de Cabrero, seguido de las poblaciones de Laja y Gomero (Fig. 1E). Tal resultado evidencia que la especie posee una estrategia diferencial en el almacenamiento del agua dependiendo del área en donde se encuentra (Nobel \& Bobich 2002). La mayor suculencia de los individuos de Cabrero podría estar relacionada con la fuerte estacionalidad de esta región (Hoffmann 1995, Mourgues 1982), caracterizada por presentar veranos muy largos y secos y precipitaciones concentradas en el período del invierno. En consecuencia, los individuos requerirían de tallos más suculentos para almacenar el agua líquida para su utilización en periodos de escasez (Cornejo \& Simpson 
1997), y así poder mantener su actividad metabólica, el crecimiento y la productividad durante los meses más secos del verano (Turner \& Jones 1980, Hanson \& Hitz 1982). En contraste, en Laguna del Laja, durante el verano, la temperatura media mensual puede llegar a los $13,6{ }^{\circ} \mathrm{C}$ (enero) (Mourgues 1982). Con el aumento en la temperatura, ocurren deshielos, que probablemente podrían proporcionar una mayor disponibilidad de agua para los individuos de $M$. poeppigii, los que no necesitarían almacenarla.

En este estudio encontramos que los atributos anatómicos de $M$. poeppigii varían fuertemente de acuerdo con la posición de las poblaciones en el gradiente de altitud de la zona centro-sur de Chile, y que esta variación podría estar relacionada con los factores ambientales particulares a los que se encuentra sometida cada población estudiada. El hecho de que esta especie se distribuya tan australmente (Saldivia \& Rojas 2008), podría ser reflejo de individuos altamente plásticos, tolerantes al estrés causado por el frío (Ladjal et al. 2005, Körner 2003). Además, los cambios anatómicos que esta especie viene experimentando a lo largo del gradiente de altitud podrían derivar en la formación de ecotipos, localmente adaptados a las condiciones de sus hábitats y sus variaciones. Sin embargo, se necesita evaluar más a profundidad esta posibilidad, tanto del punto de vista morfológico como genético, para inferir con mayor exactitud si el rango de distribución de esta cactácea estaría determinado por un agregado total de poblaciones localmente adaptadas.

\section{AGRADECIMIENTOS}

Los autores agradecen a Matías Pérez, Kattia Palacio y Fernando Carrasco por su ayuda en el trabajo de terreno. A Evelyn Rubilar, por su ayuda con los cortes anatómicos de Maihuenia poeppigii. Al Dr. Marcelo Baeza por proporcionar el equipo fotográfico. A la Dra. Nicol Fuentes, por sus comentarios a versiones previas de este manuscrito.

\section{BIBLIOGRAFÍA}

Alberdi, M. \& C. Donoso. 2004. Variabilidad en Embothrium coccineum. En: C. Donoso, A. Premoli, L. Gallo \& R. Ipinza (eds.), Variación intraespecífica en las especies arbóreas de los bosques templados de Chile y Argentina, pp. 345-356. Editorial Universitaria, Santiago, Chile.

ACKerLy, D. 2004. Functional strategies of chaparral shrubs in relation to seasonal water deficit and disturbance. Ecological Monographs 74: 25-44.

Alves, E. \& A. Angyalossy. 2001. Ecological trends in the wood anatomy of some Brazilian species. 1. Growth rings and vessels. IAWA Journal 21: 31-40.

Arias, S. \& T. Terrazas. 2001. Variación en la anatomía de la madera de Pachycereus pecten-aboriginum (Cactaceae).
Anales del Instituto de Biología, Universidad Nacional Autónoma de México. Série Botánica 72(2): 157-169.

Arnold, D.H. \& J.D. Mauseth. 1999. Effects of environmental factors on development of wood. American Journal of Botany 86: 367-371.

Barajas-Morales, J. 1985. Wood structural differences between trees of two tropical forests. IAWA Bulletin NS 6: 355-364.

BAUERT, M.R. 1996. Genetic Diversity and ecotypic differentiation in artic and alpine populations of Polygonum viviparum. Artic and Alpine Research 28: 190-195.

CABrera, H.M. 2002. Respuestas ecofisiológicas de plantas en ecosistemas de zonas con clima mediterráneo y ambientes de alta montaña. Revista Chilena de Historia Natural 75: 625-637.

Carlquist, S. 1975. Ecological strategies of xylem evolution. University of California Press, Los Angeles. 259 pp.

Carlquist, S. \& D.A. Hoekman. 1985. Ecological wood anatomy of the woody southern Californian flora. IAWA Bulletin NS 6: 319-347.

Cavieres, L.A., A. Peñaloza \& M.T.K. Arroyo. 2000. Altitudinal vegetation belts in the high Andes of central Chile. Revista Chilena de Historia Natural 73: 331-344.

Cavieres, L.A., M.T.K. Arroyo, A. Peñaloza \& M.A. MolinaMontenegro. 2002. Nurse effect of Bolax gummifera (Apiaceae) cushion plants in the alpine vegetation of the Chilean Patagonian Andes. Journal of Vegetation Science 13: 547-554.

Cavieres, L.A., E.I. Badano, A.S. Almeida, S.G. González \& M.A. Molina-Montenegro. 2006. Positive interactions between alpine plant species and the nurse cushion plant Laretia acaulis do not increase with elevation in the Andes of central Chile. New Phytologist 169: 59-69.

Cornelissen, J.H.C., S. Lavorel, E. Garnier, S. Díaz, N. Buchmann, D.E. Gurvich, P.B. Reich, H. ter Steege, H.D. Morgan, M.G.A. van der Heidden, J.G. Pausas \& H. POORTER. 2003. A handbook of protocols for standardised and easy measurement of plant functional traits worlwide. Australian Journal Botany 51: 335- 380.

Cornejo, D.O. \& B.B. Simpson. 1997. Analysis of form and function in North American columnar cacti (tribe Pachycereeae). American Journal of Botany 84: 1482-1501.

Davis, S.D., J.S. Sperry \& U.G. Hacke. 1999. The relationship between xylem conduit diameter and cavitation caused by freezing. American Journal of Botany 86(10): 1367-1372.

Di castri, F. \& E.R. HajeK. 1976. Bioclimatología de Chile. Editorial Universidad Católica de Chile, Santiago. 128 pp.

EwERS, F.W. 1985. Xylem structure and water conduction in conifer trees, dicot trees, and lianas. IAWA Bulletin new series 6: 309-371

EwERs, F.W. \& J.B. FISHER. 1989. Techniques for measuring vessel lengths and diameters in stems of woody plants. American Journal of Botany 76: 645-656.

EwErs, F.W. \& J.B. Fisher. 1991. Why vines have norrow stems: histological trends in Bahuinia (Fabaceae). Oecología 8: 233-237.

FAHN, A., E. Werker \& P. BAAS. 1986. Wood anatomy and identification of trees and shrubs from Israel and adjacent regions. The Israel Academy of Sciences and humanities, Jerusalem. 221 pp.

Falster, D.S. \& M. Westoby. 2005. Alternative height strategies 
Variación de atributos anatómicos en Maihuenia poeppigii: CABRAL, T. ET AL.

among 45 dicot rain forest species from tropical queensland, Australia. Journal of Ecology 93: 521-535.

Filella, I. \& J. Peñuelas. 1999. Altitudinal differences in UV absorvence, UV reflectance and related morphological traits of Quercus ilex and Rhododendron ferrugineum in the Mediterranean region. Plant Ecology 145: 157-165.

Galen, C., J.S. Shore \& H. Deyoe. 1991. Ecotypic divergente in alpine Polemonium viscosum: genetic structure, quantitative variation and local adaptation. Evolution 45: 1218-1228.

GARTNER, B. 1995. Plant Stems: physiology and functional ecology. Academic Press, London. 440 pp.

Gartner, B., S. Bullock, H. Mooney, B. Brown \& J. Whitbeck. 1990. Water transport of vine and tree stems in tropical deciduous forest. American Journal of Botany 77: 742749 .

Gibson, A.C. 1973. Comparative anatomy of secondary xylem in Cactoideae (Cactaceae). Biotropica 5: 29-65.

Hacke, U.G. \& J.S. Sperry. 2001. Functional and ecological xylem anatomy. Perspectives in Plant Ecology, Evolution and Systematics 4: 97-115.

Hanson, A.D. \& W.D. Hitz. 1982. Metabolic responses of mesophytes to plant water deficits. Annual Review of Plant Physiology 33: 163-203.

Hijmans, R.J., S.E. Cameron, J.L. Parra, P.G. Jones \& A. Jarvis. 2005. Very high resolution interpolated climate surfaces for global land areas. International Journal of Climatology 25: 1965-1978.

Hoch, G., M. Popp \& C. Körner. 2002. Altitudinal increase or mobile carbon pools in Pinus cembra suggest sink limitation of growth at the Swiss treeline. Oikos 98: 361374.

Hoffmann, A. 1995. Flora silvestre de Chile, zona central. Ed. Fundación Claudio Gay, Santiago. 254 pp.

Hoffmann, A. \& H.E. Walter. 2004. Cactáceas en la flora silvestre de Chile. Ed. Fundación Claudio Gay, Santiago. 307 pp.

Jacobsen, A., F.W. Ewers, R.B. Pratt, W.A. Paddock III \& S.D. DAvis. 2005. Do xylem fibers affect vessel cavitation resistance? Plant Physiology 139: 546-556.

JimÉnez-CAStilLo, M. \& C.H. Lusk. 2013. Vascular performance of woody plants in a temperate rain forest: lianas suffer higher levels of freeze-thaw embolism than associated trees. Functional Ecology 27: 403-412.

Kahn-Jetter, Z., L.S. Evans \& S. Butwell. 2002. Stresses on joints of Opuntia laevis: forces necessary for mechanical failure and role of lignified xylem cells as resistance components. Environmental and Experimental Botany 47: 231-237.

KörnER, C. 1999. Alpine plant life: Functional plant ecology of high mountain ecosystems. Springer, Berlín. 338 pp.

KöRner, C. \& W. LARCher. 1988. Plant life in cold climates. In: S.F. Long \& F.I. Woodward (eds.), Plants and temperature, pp. 25-47. Cambridge University Press, Cambridge, UK.

Körner, C. 2003. Alpine Plant Life. Springer-Verlag, Berlin, Alemania. $359 \mathrm{pp}$.

Kraus, R.G. 1993. Ökophysiologie chilenischer Kakteen. Dr. Rer. Nat. Dissertation. Institut für Botanik und Mikrobiologie der Technischen Universität München, Deutschland. $301 \mathrm{pp}$.

Krebs, C.J. 1985. Ecología: Estudio de la distribución y la abundancia, 2da edición. Editorial Harla. Ciudad de
México. 753 pp.

Ladjal, M., H.U.C. Roland \& M. Ducrey. 2005. Drought effects on hydraulic conductivity and xylem vulnerability to embolism in diverse species and provenances of Mediterranean cedars. The Physiologist 25: 1109-1117.

Lambers, H., F.S. Chapin \& T.L. Pons. 1998. Plant physiological ecology. Springer-Verlag, New York, New York, 540 pp.

Larcher, W. 2012. Biotic temperatures in the high Alps. In: C. Lütz (ed.), Plants in Alpine Regions, pp. 21-27. Springer, Vienna, Austria.

Leuenberger, B.E. 1992. Observations in Maihuenia (Cactaceae) in Argentina and Chile. Part 1: Introduction and notes on root systems. Cactus Succulent Journal 64: 71-79.

Leuenberger, B.E. 1997. Maihuenia- monograph of Patagonian genus of Cactaceae. Botanische Jahrbücher Systematik 119(1): 1-92.

Lindorf, H. 1994. Eco-anatomical wood features of species from a very dry tropical forest. IAWA Journal 15: 361-376.

Luebert, F. \& P. Pliscoff. 2006. Sinopsis bioclimática y vegetacional de Chile. Editorial Universitaria. Santiago de Chile. 316 pp.

Maherali, H., W.T. Pockman, \& R.B. Jackson. 2004. Adaptive variation in the vulnerability of woody plants to xylem cavitation. Ecology 85: 2184-2199.

Mark, A.F., S. Porter, J.J. Piggott, P. Michel, T. Maegli \& K.J.M. Dickinson. 2008: Altitudinal patterns of vegetation, floras, life forms, and environments in the alpine zone of the Fiord Ecological Region, New Zealand. New Zealand Journal of Botany 48: 205-237.

Mauseth, J.D. 1993. Water-storing and cavitation-preventing adaptations in wood of cacti. Annals of Botany 72:81-89.

Mauseth, J.D. 1999. Anatomical adaptations to xeric conditions in Maihuenia (Cactaceae), a relictual, leaf-bearing cactus. Journal of Plant Research 112: 307-315.

Mauseth, J.D. 2000. Theoretical aspects of surface-to-volume ratios and water-storage capacities of succulent shoots. American Journal of Botany 88: 1107-1115.

Mauseth, J.D. 2006. Structure-Function Relationships in Highly Modified Shoots of Cactaceae. Annals of Botany 98: 901926.

Mourgues, V.S.C.H. 1982. Plan de manejo Parque Nacional Laguna del Laja. Documento interno. CONAF VIII Región. Concepción, Chile. 211 pp.

Neuffer, B. \& S. Bartelheim. 1989. Genecology of Capsella bursa-pastoris from an altitudinal transect in the Alps. Oecology 81: 521-527.

Nobel, P.S. \& E.G. Boвich. 2002. Environmental Biology. In: PS. Nobel (ed.), Cacti biology and uses, pp. 61-62. University of California Press, Berkeley, Los Angeles, USA.

Pate, J.S. \& M.J. CANNY. 1999. Quantification of vessel embolisms by direct observation: a comparison of two methods. New Phytologist 141: 33-44

Preston, K.A., W.K. Cornwell \& J.L. Denoyer. 2006. Wood density and vessel traits as distinct correlates of ecological strategy in 51 California coast range angiosperms. New Phytologist 170(4): 807-818.

Rochow, T.F. 1970. Ecological investigations of Thlaspi alpestre L. along an elevational gradient in the central Rocky Mountains. Ecology 51: 649-656.

Rundel, P., A. Smith \& F. Meinzer (Eds). 1994. Tropical Alpine 
Environments. Cambridge University Press, UK. 376 pp.

SAKAI, A. \& W. LARChER. 1987. Frost Survival of Plants. Responses and adaptation to Freezing Stress. Ecological Studies.Vo1 62. Springer-Verlag, Berlin. 321 pp.

Saldivia, P. \& G. Rojas. 2008. Nuevos registros y antecedentes de la familia Cactaceae para Chile en la Región de Aisén. Gayana Botánica 65(2): 198-208.

SÁnchez-Díaz, M. \& J. Aguirreolea. 2000. Transporte de agua y balance hídrico en la planta. En: J. Azcón \& M. Talón (eds.), Fundamentos de fisiología vegetal, pp. 45-64. McGraw Hill Interamericana. Barcelona. España.

Santiago, L.S., G. Goldstein, F.C. Meinzer, J.B. Fisher, K. Machado, D. Woodruff \& T. Jones. 2004. Leaf photosynthetic traits scale with hydraulic conductivity and wood density in Panamanian forest canopy trees. Oecologia 140: 543-550.

SChnitzer, S.A. \& F.A. Bongers. 2002. The ecology of lianas and their role in forests. Trends in Ecology \& Evolution 17: 223-230.
Souto, C.P., A.C. Premoli \& P.B. Reich. 2009. Complex bioclimatic and soil gradients shape leaf trait variation in Embothrium coccineum (Proteaceae) among southern forests in Patagonia. Revista Chilena de Historia Natural 82: 209-222.

Terrazas, T.S. \& J.D. Mauseth. 2002. Shoot Anatomy and Morphology. In: P.S. Nobel (ed.), Cacti biology and uses, pp. 23-37. University of California Press, Berkeley, Los Angeles, USA.

Turner, N.C. \& M.M. Jones. 1980. Turgor maintenance by osmotic adjustment: a review and evaluation. In: N.C. Turner \& P.J. Kramer (eds.), Adaptation of plants to water and high temperature stress, pp. 87-103. John Wiley \& Sons, London, UK.

Zimmermann, M.H. 1983. Xylem structure and the ascent of sap. Springer-Verlag, Berlín, Alemania. 284 pp.

Zimmermann, M.H. \& C.L. Brown. 1971. Trees. Structure and function. Springer- Verlag. New York, USA. 336 pp.

Recibido: 23.10 .15

Aceptado: 09.03.16 